\title{
Wissenschaftliche Politikberatung für gute Dienstleistungsarbeit
}

\section{Eine Einordnung der Tagung „Dienstleistungsforschung und Dienstleistungspolitik“ im April 2013 in Düsseldorf}

Melanie Diermann, Dorothea Voss

\section{Kernaussagen}

Das Feld der Dienstleistungsarbeit ist heterogen und erstreckt sich über sehr unterschiedliche Branchen und Berufsgruppen. Arbeitnehmerinteressen werden in diesem Kontext von unterschiedlichen gewerkschaftlichen Akteuren vertreten, sodass der gemeinsame Abstimmungsbedarf groß ist. Die von der Hans-Böckler-Stiftung und ver.di organisierte Dienstleistungstagung 2013 hat vor diesem Hintergrund den Auftakt geliefert, die strategische Diskrepanz zwischen branchenspezifischen Einzelkonzepten einerseits und der Notwendigkeit einer abgestimmten Vorgehensweise in politischen Diskursen andererseits zu überwinden.

\section{Einleitung}

Das Angebot von Lärmschutzmaßnahmen für Menschen, die in lärmintensiven Umgebungen arbeiten, ist eine wichtige Errungenschaft. Sie ging grundlegend aus der wissenschaftlich belegten Erkenntnis hervor, dass Prävention zur Erhaltung der Arbeitskraft beiträgt und insofern nicht nur wertethisch richtig sonder auch wirtschaftlich vorteilhaft ist. Bauarbeiter die neonfarbene Ohrstöpsel tragen, während sie mit Presslufthämmern Beton bearbeiten, sind das Ergebnis dieses Erkenntnisprozesses. Wer einmal eine vollbesetzte Kindergartengruppe besucht hat, weiß, dass auch Erzieherinnen einem ausgesprochen hohen Lärmpegel ausgesetzt sind. Und wer darüber nachdenkt, wie geeignete Lärmprävention in ihrem Fall aussehen könnte, kommt unmittelbar zu der Erkenntnis, dass das Verteilen von Ohrstöpseln hier nicht als Problemlösung geeignet ist. Das Beispiel illustriert die Notwendigkeit, Dienstleistungsarbeit aus dem Kontext der traditionellen, an Industriearbeit orientierten Forschung herauszulösen und im Lichte dienstleistungsspezifischer Anforderungen zu erörtern. Vor diesem Hintergrund standen Dienstleistungsforschung und Dienstleistungspolitik im Zentrum einer Tagung, zu der die Hans-Böckler-Stiftung in Kooperation mit der Dienstleistungsgewerkschaft ver.di am 24. und 25. April 2013 in Düsseldorf eingeladen hatte. Die Veranstaltung richtete sich an Interessierte aus Wissenschaft, Gewerkschaften, Unternehmen und Politik. Ziel der Veranstalter war es, den „state of the art“ der Dienstleistungsforschung zu präsentieren und auf dieser Basis Handlungsempfehlungen für eine "gute" Dienstleistungspolitik abzuleiten. ${ }^{1}$ Ziel dieses Beitrages ist es, wesentliche Ergebnisse der Tagung festzuhalten und in den Kontext wissenschaftlicher und gewerkschaftlicher Politikberatung zu stellen. $^{2}$

\section{Gewerkschaftliche Akteurskonstellation und Hintergrund der Tagung}

Das Feld der Dienstleistungen umfasst eine große Spannweite an unterschiedlichen Tätigkeiten in unterschiedlichen Branchen und Berufen: Bankdienstleistungen fallen in diesen Bereich, Tätigkeiten in der Pflege und im Erziehungswesen, aber auch die Beratung und Entwicklung in Industriebranchen oder im Informations- und Telekommunikationssektor. Etwa zwei Drittel aller in Deutschland sozialversicherungspflichtig Beschäftigten üben gegenwärtig Dienstleistungstätigkeiten aus (Bundesagentur für Arbeit 2013). Die Interessen dieser Beschäftigten werden in politischen Diskursen von den für Dienstleistungsbranchen zuständigen Gewerkschaften vertreten. Neben den Dienstleistungsgewerkschaften sind das insbesondere auch die Industriegewerkschaften, in deren Vertretungsbereich die so genannten industrienahen Dienstleistungen fallen - davon ist die Rede, wenn etwa der VW-Konzern ein Drittel seines Umsatzes im Unternehmensbereich Finance mit klassischen Bankdienstleistungen und insofern nicht mehr mit dem industriellen Aspekt „Autos zu verkaufen“ erwirtschaftet.

Indem mehrere Gewerkschaften involviert sind, ist der gemeinsame Abstimmungs- und Strategiefindungsbedarf im Kontext von Dienstleistungsforschung und Dienstleistungspolitik von großer Bedeutung. Die Motivation zur Teilnahme an der Tagung war insofern für Gewerkschaftler, Wissenschaftler und Praktiker gleichermaßen die Überzeugung, dass ein Konsens über übergeordnete (strategische) Ziele für gewerkschaftliches Handeln notwendig ist. Die Hans-BöcklerStiftung nimmt im Kontext dieses Abstimmungsprozesses eine moderierende Rolle ein, um ihrem Satzungszweck gemäß Aspekte der „Theorie und Praxis der Arbeitnehmerinteressenvertretung durch wissenschaftliche Beratung und Qualifizierung “ zu fördern. Gewerkschaftliche Akteure sind dabei für die Stiftung grundlegend auf zwei Ebenen eine wichtige Zielgruppe: Zum einen repräsentieren sie Arbeitnehmerinteressen

1 Für ver.di bestand das zentrale Anliegen der Tagung darin, eine Initiative für „Gute Arbeit für gutes Geld und sichere Arbeitsverhältnisse“ zu ergreifen. Die wesentlichen Punkte fasst ein ver.di-Hintergrundpapier zusammen (ver.di 2013).

2 Die Tagungsdokumentation ist unter folgendem link zu finden: http:// boeckler.de/28733_41975.htm. 
in unternehmens-, berufsgruppen- und branchenspezifischen Kontexten und werden in dieser Funktion von der Stiftung beraten. Zum anderen werden Gewerkschaften von der Stiftung in ihrer Funktion als Vertreterinnen von Arbeitnehmerinteressen in politischen Diskursen beraten. Politikberatung erfolgt auf dieser Basis entlang von Fakten, die wissenschaftliche Studien ergeben haben, und durch die Schaffung entsprechender Diskussionsforen.

Die Tagung war vor diesem Hintergrund darauf ausgelegt, grundlegende Gestaltungsansätze für gute Dienstleistungsarbeit auf der strategischen Ebene herauszuarbeiten und branchenübergreifend Erkenntnisse, künftige Fragestellungen und politische Forderungen $\mathrm{zu}$ formulieren. Wertschätzung, soziale Sicherung und angemessene Bezahlung sind in diesem Kontext wesentliche Aspekte. Der Zugang, Dienstleistungsarbeit auf der strategischen (also berufsgruppen- und branchenübergreifenden) Ebene in Betracht zu nehmen um wesentliche Ziele als übergeordnete Leitstrategie festzulegen, kann als innovativ bezeichnet werden, da sich Ansätze zur Formulierung von Dienstleistungspolitik bisher vor allem entlang berufs- und branchenspezifischer und damit sehr konkreter Fragestellungen orientiert haben.

\section{Dienstleistungsforschung: Ansätze und Forschungsfragen}

Wissenschaft ist stets darauf ausgelegt, gesellschaftliche Entwicklung zu berücksichtigen und zu erklären. Forscher sind dann ihrer Zeit voraus, wenn es ihnen gelingt, Fragen und Spannungsfelder zu identifizieren und Lösungspfade zu entwerfen, bevor sie offensichtlich oder problematisch werden. Der Paradigmenwechsel vom industriell geprägten Zeitalter hin zur Dienstleistungsgesellschaft ist als ein solches Spannungsfeld zu bezeichnen und auch entsprechend wissenschaftlich reflektiert worden. Jedoch kann von einer etablierten und eigenständigen Disziplin der Dienstleistungswissenschaft oder auch „Service Science“ derzeit nicht gesprochen werden (Birken/Dunkel 2013). Vielmehr sucht die multimethodisch und interdisziplinär ausgerichtete Dienstleistungsforschung nach Ansicht derjenigen, die sie als notwendiges wissenschaftliches Pendant zur Dienstleistungsgesellschaft sehen gegenwärtig nach ihrem Platz in der Wissenschaftslandschaft (Satzger/ Dunkel 2011, S. 473). Sie speist sich aus Wissensbeständen und den spezifischen Herangehensweisen von mindestens vier Disziplinen, nämlich den Wirtschafts- und Sozialwissenschaften, den Ingenieurswissenschaften und der Informatik.

Weitgehender Konsens besteht darüber, dass die „Beschreibung, Erklärung und Gestaltung sozio-technischer Systeme “ in den Blick genommen werden soll (Satzger/Dunkel 2011, S. 472). Sozio-technische Systeme in Dienstleistungen bzw. Dienstleistungsprozesse sind dabei vielfältig, geht es doch genauso um die Entwicklung von IT-Systemen, die Beratung in Finanzangelegenheiten, wie auch um die Pflege von Kranken, die Bildung von Kindern oder den Verkauf von Waren, um nur einige Beispiele zu nennen. Einblicke in die Vielfalt der Perspektiven von Dienstleistungsforschung gaben die Vorträge in den sechs Foren des ersten Tages, wobei gemäß der Zielsetzung der Veranstalter bei der Analyse von Dienstleistungssystemen ein Schwerpunkt auf die Auswirkungen auf Arbeit und Beschäftigung gelegt werden sollte. So wurde thematisiert, wie sich Digitalisierung und Internationalisierung auf die Organisation von Dienstleistungen in globalisierten Unternehmen und die Arbeitsbedingungen von Beschäftigten auswirken. An der Gestaltung von Dienstleistungsprozessen sind Kunden mehr oder weniger direkt beteiligt, daher - so das Thema in weiteren Foren - ist eine zentrale Perspektive von Dienstleistungsforschung die Interaktion von Menschen untereinander wie auch zwischen Mensch und Technik. Dass Wertschöpfung ohne die Wertschätzung der Beschäftigten als Dienstleistungserbringer nicht denkbar ist, wurde genauso betont, wie die Rolle der Mitbestimmung als Quelle von Innovation und auch Mahnerin für Investitionen, zum Beispiel in die Professionalisierung und Qualifizierung von Dienstleistungsbeschäftigten.

Insgesamt wurde durch die Präsentation des „state of the art“ der Dienstleistungsforschung deutlich, dass eine Gestaltung von Dienstleistungen und Dienstleistungssystemen kaum ohne eine Gestaltung von Arbeit und Beschäftigung auskommt: Menschliche Arbeit ist ein Kernelement in sozio-technischen Systemen. Wie sie eingesetzt wird, wo ungenutzte Potenziale bestehen und welche Herausforderungen für die Gestaltung von Arbeit und Beschäftigung bestehen, ist daher eine zentrale Perspektive unterschiedlicher wissenschaftlicher Disziplinen der Dienstleistungsforschung. Eine Erzieherin ist beispielsweise nicht doppelt so produktiv, wenn sie statt zehn Kindern zwanzig betreuen muss. An diesem idealtypischen Beispiel manifestiert sich die Notwendigkeit, Fragen zur Arbeitsgestaltung in einem im Vergleich zur klassischen Industrieforschung neuen Licht zu reflektieren. Hier bedarf es etwa auch neuer Indikatoren der Messbarkeit, die zeigen, worin genau sich etwa Produktivität im Bereich der gesellschaftsnahen Dienstleistungen ausdrückt und inwieweit eine maßgebliche Orientierung an betriebswirtschaftlichen Kennziffern normativ überhaupt sinnvoll oder gewollt ist.

\section{Dienstleistungspolitik: Ziele und Tagungsergebnisse}

Inwieweit sind die vorgestellten Ergebnisse der Dienstleistungsforschung nun von Bedeutung für diejenigen, die für eine zukunftsgerichtete Dienstleistungspolitik eintreten? Inwiefern kann von einer Dienstleistungstagung „impact“ in den politischen Raum hinein ausgehen? Die Zusammensetzung der Teilnehmerinnen und Teilnehmer der Tagung war heterogen: Vertreterinnen und Vertreter aus Mitbestimmungsgremien von Unternehmen nahmen ebenso teil wie aus Gewerkschaften und der Politik. Sie sind in je unterschiedliche Kontexte eingebunden und an unterschiedlichen Stellen in die Gestaltung von Dienstleistungen und damit die Politik der Dienstleistungen eingebunden.

Ziel des zweiten Tages der Tagung war es daher, Eckpfeiler einer Dienstleistungspolitik erkennbar werden zu lassen, die für die Gestaltung von „Guter Arbeit“ unerlässlich sind. Nach den Impulsen aus der Dienstleistungsforschung am ersten Tag 
wurden am zweiten Tag der Tagung Gestaltungsansätze in unterschiedlichen Politikfeldern in den Blick genommen. Anforderungen an die Bildungs-, Arbeits- und Leistungspolitik wurden formuliert, weil ausreichende Qualifikation in Verbindung mit einer betrieblichen Arbeits- und Leistungspolitik, die eine gesunderhaltende und alternsgerechte Nutzung der Arbeitskraft sicher stellt, eine Grundvoraussetzung für gute Dienstleistungen ist. Für die Gestaltung und Entwicklung von Dienstleistungen aus Nutzersicht ist auch die Lebenslaufperspektive ein fruchtbarer Ansatz, denn in einer solchen Perspektive wird erkennbar, welche Art von Dienstleistungen Menschen über den Lebenslauf benötigen, wo also Bedarf besteht. Damit ist auch die Innovationspolitik angesprochen, die unter zunehmendem Einfluss europäischer Regulierung die Rahmenbedingungen setzt für die Weiterentwicklung von Dienstleitungen.

Als Ergebnis der Tagung kann festgehalten werden, dass „alte“ Fragen nach den notwendigen Rahmenbedingungen für „Gute Arbeit“ nichts an Bedeutung eingebüßt haben. Deutlich wurde jedoch, dass sie sich unter veränderten Bedingungen im Betrieb und der Gesellschaft im Zeitalter der Dienstleistungswirtschaft immer wieder neu stellen und neue Antworten erfordern. Die Tagung hat dazu beigetragen, dass die Konturen einer zukunftsfähigen Gestaltung von Dienstleistungen und Dienstleistungsarbeit deutlicher geworden sind. Der Besuch der Ministerin für Innovation, Wissenschaft und Forschung des Landes Nordrhein-Westfalen, Svenja Schulze, hat dabei insbesondere auch die gehörte Forderung unterstrichen, dass es einer intensiveren öffentlich geförderten Dienstleistungsforschung bedarf, die über das bloße Erforschen von Dienstleistungsarbeit hinausgehen muss.

\section{Fazit und Ausblick}

Dienstleistungen tragen einen wachsenden Anteil an der Wertschöpfung in Deutschland. Angesichts der technischen und arbeitsorganisatorischen Möglichkeiten, die sich unter anderem aus Innovationen in der Informations- und Kommunikationstechnik ergeben, verändern sich Tätigkeiten und Arbeitsbedingungen für eine Mehrheit der Beschäftigten. Gleichzeitig erwachsen aus den veränderten Arbeits- und Lebensbedingungen neue Anforderungen an Dienstleistungen. Vor diesem Hintergrund war es das Ziel der Veranstalter, im Kontext der Tagung „Dienstleistungsforschung und Dienstleistungspolitik" aktuelle wissenschaftliche Erkenntnisse zu präsentieren und Impulse für eine „gute“ Dienstleistungspolitik zu setzen.

Wissenschaftliche Politikberatung findet dabei auf zwei Ebenen statt: zum einen durch die Finanzierung von Studien, und zum anderen durch das Schaffen von Foren zur Diskussion und Implementierung ihrer Ergebnisse. Ein wichtiger Hebel zur Erreichung dieser Ziele ist die akteursübergreifende Verständigung, die einer konsistenten Politikformulierung vorausgehen muss. Die maßgebliche Bedeutung dieser Verständigung wurde auch im Rahmen des Abschlussplenums der Tagung von Christiane Benner (Mitglied im Vorstand der IG Metall) und Lothar Schröder (Mitglied im Vorstand der ver.di) verdeutlicht: Sie betonten, dass unabhängig von konkreten Branchen eine der zentralen Herausforderungen für Gewerkschaften darin liege, Inhalte und Gegenstände von Dienstleistungsarbeit gewerkschaftsübergreifend zusammen zu denken, so dass gute Dienstleistungspolitik künftig gute Dienstleistungsarbeit hervorbringen kann - branchen- und berufsgruppenunabhängig. Die Weiterverfolgung der akteursübergreifenden Verständigung ist in diesem Zusammenhang ebenso von Bedeutung wie die Umsetzung der Forschungsbedarfe, die im Kontext der Tagung offensichtlich geworden sind. Vor diesem Hintergrund stellt sich auch die Frage, inwieweit das Format „Dienstleistungstagung“ in drei Jahren erneut aufgerufen werden kann, um Fortschritte und neue Entwicklungsbedarfe festzustellen.

\section{Literatur}

Birken, T. \& W. Dunkel (2013). Dienstleistungsforschung und Dienstleistungspolitik. Hans-Böckler-Stiftung, Arbeitspapier 282. Düsseldorf.

Bundesagentur für Arbeit (2013). Beschäftigtenstatistik, http://statistik.arbeitsagentur.de/Navigation/Statistik/Statistik-nach-Themen/ Beschaeftigung/Beschaeftigung-Nav.html [abgerufen am 26. 05. 2013].

Satzger, G. \& W. Dunkel (2011). Service Science - Potenziale für die Weiterentwicklung der Dienstleistungsgesellschaft. In WSI Mitteilungen 9/2011. S. 470-477.

Hans-Böckler-Stiftung (2013). Dokumentation der Tagung „Dienstleistungsarbeit und Dienstleistungsforschung “, http://www.boeckler.de/ 28733_41975.htm [abgerufen am 20.5.2013].

Ver.di (2013). Hintergrundpapier der Dienstleistungsgewerkschaft ver.di zur Tagung. www.boeckler.de/pdf/v_2013_04_24_25_verdi.pdf [abgerufen am 20.5.2013].

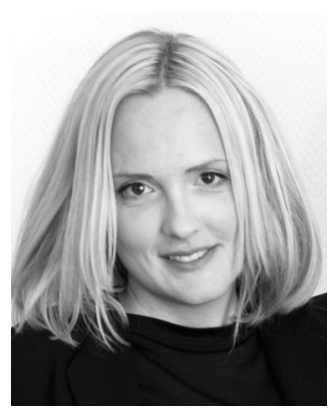

Dr. Melanie Diermann ist in der Abteilung Forschungsförderung der Hans-Böckler-Stiftung zuständig für Politikvermittlung und Transfer. Sie studierte Politikwissenschaft, Psychologie sowie Kommunikationsund Medienwissenschaften an der Universität Duisburg-Essen, es folgte die Promotion an der NRW School of Governance. Email: melanie-diermann@boeckler.de.

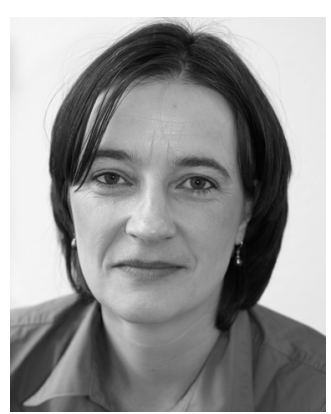

Dr. Dorothea Voss ist Referatsleiterin des Forschungsschwerpunkts „Zukunft des Sozialstaats/Sozialpolitik" der Hans-Böckler-Stiftung. Sie studierte Volkswirtschaftslehre, Soziologie und Politikwissenschaften an der Universität zu Kiel und am Institut für Weltwirtschaft und wurde an der Universität DuisburgEssen promoviert. Email: dorotheavoss@boeckler.de. 